\title{
Oral fluoroquinolone use and serious arrhythmia: bi-national cohort study
}

\author{
Malin Inghammar, ${ }^{1}$ Henrik Svanström, ${ }^{2}$ Mads Melbye, ${ }^{2}$ Björn Pasternak, ${ }^{2}$ Anders Hviid ${ }^{2}$
}

'Lund University, Department of Clinical Sciences Lund, Section

for Infection Medicine, Lund,

Sweden

2Department of Epidemiology Research, Statens Serum Institut, 2300 Copenhagen S, Denmark

Correspondence to: M Inghammar malin.inghammar@med.lu.se

Additional material is published online only. To view please visit the journal online.

Cite this as: $B M$ J 2016;352:i843 http://dx.doi.org/10.1136/bmj.i843 Accepted: 29 January 2016

\section{ABSTRACT}

OBJECTIVE

To evaluate if oral fluoroquinolone use is associated with an increased risk of serious arrhythmia.

\section{DESIGN}

Bi-national cohort study, linking register data on filled prescriptions, cases of serious arrhythmia, and patient characteristics.

SETTING

Denmark, 1997-2011; Sweden, 2006-13.

\section{PARTICIPANTS}

The study cohort was derived from a source population of all Danish and Swedish adults, aged 40 to 79 years. 909656 courses of fluoroquinolone use (ciprofloxacin $82.6 \%$, norfloxacin $12.1 \%$, ofloxacin $3.2 \%$, moxifloxacin $1.2 \%$, and other fluoroquinolones $0.9 \%$ ) and 909656 courses of penicillin $\mathrm{V}$ use, matched 1:1 on propensity score, were included.

\section{MAIN OUTCOME MEASURE}

The main outcome was risk of serious arrhythmia (fatal and non-fatal), comparing courses of fluoroquinolone use with courses of penicillin $V$ use (an antibiotic with no pro-arrhythmic effect). The risk period of interest was current use, defined as days 0-7 of treatment. Subgroup analyses were conducted according to country, sex, age, underlying cardiovascular disease, concomitant use of drugs known to increase the risk of torsades de pointes, fluoroquinolone type, and levels of arrhythmia risk score.

\section{RESULTS}

144 cases of serious arrhythmia occurred during follow-up, 66 among current fluoroquinolone users (incidence rate 3.4 per 1000 person years) and 78 among current penicillin users (4.0 per 1000 person years); comparing oral fluoroquinolone treatment with penicillin $\mathrm{V}$, the rate ratio was 0.85 (95\% confidence interval 0.61 to 1.18). Compared with penicillin V, the absolute risk difference was -13 (95\% confidence interval -35 to 16 ) cases of serious arrhythmia per

\section{WHAT IS ALREADY KNOWN ON THIS TOPIC}

Fluoroquinolones are weak inhibitors of the cardiac potassium channels and may cause prolongation of the QT interval

Whether this translates into a risk of cardiac arrhythmia in the clinical setting is not clear

\section{WHAT THIS STUDY ADDS}

Current use of oral fluoroquinolones was not associated with increased risk of serious arrhythmia in the general adult population of two Scandinavian countries This bi-national study was large, allowing substantial statistical power, and utilised a comparative design (fluoroquinolones versus penicillin $\mathrm{V}$ ) and propensity score matching, thereby limiting the potential for confounding

1000000 courses of fluoroquinolones. The risk of serious arrhythmia was not statistically significantly increased in any of the subgroups, including analyses by fluoroquinolone type.

\section{CONCLUSIONS}

Contrary to previous reports, oral fluoroquinolone treatment was not associated with an increased risk of serious arrhythmia in the general adult populations of Denmark and Sweden. Given the statistical power of the study, even small increases in relative and absolute risk could be ruled out. Since ciprofloxacin was the most commonly used fluoroquinolone in our study, we cannot exclude that intraclass differences influence the risk of serious arrhythmia associated with other less frequently used fluoroquinolones.

\section{Introduction}

Fluoroquinolones are a class of antibiotics widely used to treat common infections, such as febrile urinary tract and lower respiratory tract infections. Although fluoroquinolones are generally well tolerated, treatment has been associated with prolongation of the QT interval. ${ }^{1}$ Owing to case reports of a possible association with sudden cardiac death, the fluoroquinolones sparfloxacin and grepafloxacin were withdrawn from the market in 1999 and 2001, respectively. ${ }^{12}$ Although this association has not been backed up by results from clinical trials due to a lack of the statistical power needed to study rare adverse events such as serious arrhythmia, ${ }^{3-8}$ a few observational studies have been published that report up to a threefold increased risk associated with fluoroquinolones. ${ }^{9-12}$ However, it cannot be ruled out that the observed increased risk was attributable to factors associated with the acute infection itself or to confounding as a result of poorer baseline health of the fluoroquinolone users in these studies.

The fluoroquinolone molecules are blockers of the human ether-à-go-go related gene (HERG) that encodes the rapid component of the cardiac potassium channel, resulting in accumulation of potassium within myocytes. Blocking of HERG can delay repolarisation (reflected in prolongation of the QT interval) and give rise to torsades de pointes, a potentially lethal arrhythmia. ${ }^{13}$ However, fluoroquinolones are weak inhibitors of the cardiac potassium channel compared with other drugs with specific inhibitory mechanisms, ${ }^{1}$ and the association between prolongation of the QT interval and torsades de pointes is complex and depends on concurrent risk factors-eg, genetic predisposition, female sex, age, underlying heart disease, and co-medication with drugs that are known to increase the risk of torsades de pointes. ${ }^{14} 15$ Thus, it remains to be determined if the risk of prolongation of the QT interval 
translates into an increased risk of serious arrhythmia in a general clinical setting.

In this large cohort study, we investigated whether oral fluoroquinolone use was associated with an increased risk of serious arrhythmia. We used a comparative study design to minimise confounding by indication, and used penicillin $\mathrm{V}$ (phenoxymethylpenicillin), an antibiotic without proarrhythmic effects, as the comparator.

\section{Methods}

\section{Study design}

We conducted a bi-national cohort study sourced from all Danish and Swedish adults, aged 40 to 79 years, from 1 January 1997 to 31 December 2011 in Denmark and from 1 January 2006 to 31 December 2013 in Sweden, linking individual data from national healthcare registers. We estimated the incidence of serious arrhythmia (fatal and non-fatal) in a historical cohort of oral fluoroquinolone users matched on propensity scores to penicillin $\mathrm{V}$ users.

\section{Data sources}

We obtained data on filled prescriptions for oral fluoroquinolones (Anatomical Therapeutic Chemical (ATC) code J01MA) and penicillin V (ATC code J01CE02) from the national prescription registries in both countries. ${ }^{1617}$ Individual data on records of serious arrhythmia were obtained from the respective national patient registries, ${ }^{1819}$ and national registries of causes of death. ${ }^{20}$ Individual information on potential confounders-that is, demographic characteristics, concomitant drug use, healthcare use, and medical history were obtained from the national population registries and national patient and prescription registries. No values were missing. Linking the information from the databases was possible using national unique personal identification numbers, assigned to all Danish and Swedish citizens.

\section{Study cohort}

We included all filled prescriptions of oral fluoroquinolones and penicillin V in adults aged 40-79 years in Denmark and Sweden during the study periods. In both countries, penicillin V is used in, and is the recommended first line treatment for, upper and lower respiratory tract infections. Fluoroquinolones with an effect on Gram positive bacteria, such as ofloxacin, moxifloxacin, and levofloxacin, have overlapping indications with penicillin $\mathrm{V}$ for respiratory tract infections, whereas older fluoroquinolones targeting Gram negative pathogens, such as norfloxacin and ciprofloxacin, are mainly used for gastrointestinal and urinary tract infections.

Each prescription was considered a separate event, and each participant could accordingly contribute multiple courses of antibiotic use to the study. Overlapping of treatment courses was not allowed, with the latest course taking precedence for treatment status. We excluded courses from people who had been admitted to hospital or who had filled any previous antibiotic prescription within 45 days before or on the prescription (index) date. To assure adequate covariate assessment, we excluded courses from those with no other prescriptions for any drug in the two years preceding the index date. To reduce the potential of confounding we also excluded those with end stage non-cardiovascular disease or substance misuse, who may have had a high pretreatment risk of arrhythmia (see supplementary appendix for a complete list).

\section{Patient involvement}

No patients were involved in setting the research question or the outcome measures, nor were they involved in developing plans for recruitment, design, or implementation of the study. No patients were asked to advise on interpretation or writing up of results. There are no plans to disseminate the results of the research to study participants or the relevant patient community.

\section{Propensity score models}

Propensity score methods were used to control for potential confounders. ${ }^{21}$ We used logistic regression to estimate the individual propensities for fluoroquinolone use. The propensity score model included the following predictors as current at the index date: country of residence, age, sex, country of birth, region of residence, calendar period, calendar year, history of cardiac disease, cerebrovascular disease, venous thromboembolism, renal disease, respiratory disease, rheumatic disease, alcohol misuse, psychiatric disorder, number of hospital admissions and visits to an emergency department, number of visits due to cardiovascular disease in the past year, a range of concomitantly used drugs, and number of prescriptions redeemed in the past year. To account for differences in antibiotic prescription between Denmark and Sweden, we estimated the propensity scores separately in each country. The supplementary appendix provides a complete list of the predictors included in the calculation of propensity score. Using the greedy 5->1 digit matching algorithm we matched courses of oral fluoroquinolone use and penicillin V use 1:1 on the estimated propensity score. ${ }^{22} 23$ To assess the balance achieved by matching we estimated standardised differences between groups. A characteristic was considered well balanced if the standardised difference was below 0.10 .

\section{Follow-up and outcome}

In the main analysis, follow-up started on the index date and ended on the date of serious arrhythmia, hospital admission unrelated to serious arrhythmia, subsequent antibiotic prescription, death (non-arrhythmic or non-sudden deaths), emigration, age 80 years old, 45 days after start of treatment, or end of study (31 December 2011 in Denmark or 31 December 2013 in Sweden), whichever came first. In the secondary analysis of death from other causes (that is, all other causes other than the main study outcome of serious arrhythmia), we did not consider subsequent antibiotic prescription and hospital admission as criteria for censoring.

Follow-up time of was subdivided into three periods: current use (0-7 days from index date), indeterminate 
use (8-14 days), and past use (15-44 days). The period of current use reflects the actual time during treatment when an acute toxic mechanism would manifest. The period of indeterminate use was included to account for the varying length of the standard treatment recommendations for fluoroquinolones (7-14 days) and penicillin $\mathrm{V}$ (7-10 days). The incidence in periods of past use should resemble the background incidence of the outcome. An increased risk in this period would reflect a delayed toxic mechanism or residual confounding from baseline differences in health between treatment groups. We assumed that if an acute toxic mechanism were true, it would manifest as an increased risk in the period of current use and disappear in periods of past use.

The outcome "serious arrhythmia” (fatal or non-fatal) was defined as a diagnosis of ventricular tachycardia or flutter (international classification of diseases, 10th revision (ICD-10) codes: I472, I490, I493), cardiac arrest (ICD-10: I460, I461, I469), or sudden death (ICD-10: R960, R961) recorded either in the patient registriesthat is, registered during a visit to an emergency ward or hospital inpatient admission, or in the cause of death registries. In the secondary analyses of fatal events and death from other causes, outcomes were based on ICD codes recorded in the cause of death registries only.

We estimated mortality from any cause other than serious arrhythmia to assess residual confounding, since statistically significant differences would indicate differences in disease severity or differential health status at baseline.

\section{Arrhythmia risk score}

We used a logistic regression model, including all potential confounders as predictors, to estimate a risk score for the outcome serious arrhythmia for subgroup analysis (see table 1), and an indicator variable for the treatment received. To calculate the risk score for each treatment course we multiplied the regression coefficients obtained from the logistic regression model by the respective covariate value at the time of starting treatment for all included factors but the treatment indicator variables, which were all set to $0 .{ }^{24}$ We then categorised all courses into three groups according to the score's distribution (in 10ths): low (1-5), moderate (6-8), and high (9-10).

\section{Statistical analyses}

Using Poisson regression, we estimated rate ratios comparing the risk of serious arrhythmia (fatal and non-fatal cases), fatal serious arrhythmia, and death due to other causes with oral fluoroquinolones use versus penicillin $\mathrm{V}$ use. We used generalised estimating equations to account for the possibility of dependency between multiple antibiotic courses in the same individual. We calculated the absolute risk difference as risk per 1000000 treatment courses: (rate ratio-1) $\times$ crude rate among users of penicillin $\mathrm{V}$.

We also estimated rate ratios in subgroups of participants classified according to country of residence, age, sex, underlying cardiovascular disease, concomitant use of drugs known to increase the risk of torsades de pointes, ${ }^{15}$ type of fluoroquinolone, and levels of arrhythmia risk score. Homogeneity of estimates across subgroups was assessed using likelihood ratio tests. We also performed sensitivity analyses using only the first episode of antibiotic treatment in each individual. All statistical tests were two sided, and we considered 95\% confidence intervals that did not overlap 1.0 and $\mathrm{P}$ values less than 0.05 to be statistically significant. Analyses were performed using SAS version 9.4 (SAS Institute).

\section{Results \\ Cohort}

Figure 1 shows the flow of cohort selection. During the study period we identified 2124632 courses of oral fluoroquinolone use (795355 in Denmark; 1329277 in Sweden) and 11065195 courses of penicillin V use (7883023 in Denmark; 3182172 in Sweden). The inclusion criteria were met for 921728 courses of oral fluoroquinolone use (309995 in Denmark; 611733 in Sweden) and 7570585 courses of oral penicillin V use (5385319 in Denmark; 2185266 in Sweden). After propensity score estimation and matching in a 1:1 ratio, the study cohort consisted of 909656 courses of oral fluoroquinolones and penicillin V use, respectively (309995 in Denmark; 599661 in Sweden). Supplementary tables s4 and s5 show the baseline characteristics of the unmatched cohorts. Before matching, the group treated with fluoroquinolones was slightly more likely to have a history of cardiovascular, respiratory, and renal disease than the penicillin V group. Users of fluoroquinolones were also more likely to have a recent history of visits to an emergency department and more likely to have a higher total number of prescribed drugs. After matching, however, the baseline characteristics were well balanced between the matched groups (table 1). Supplementary table s3 provides the odds ratios for the propensities for fluoroquinolone use for all variables.

Most of the treatment courses with oral fluoroquinolones were with ciprofloxacin $(82.6 \%)$, followed by norfloxacin (12.1\%), ofloxacin (3.2\%), moxifloxacin (1.2\%), and other fluoroquinolones $(0.9 \%)$.

\section{Main results}

A total of 429 cases of serious arrhythmia occurred, including144 during the period of current use (66 in fluoroquinolone users, 78 in penicillin $\mathrm{V}$ users). The incidence rates were 3.4 and 4.0 per 1000 person years, respectively, corresponding to a rate ratio of 0.85 (95\% confidence interval 0.61 to1.18) for oral fluoroquinolones compared with penicillin V. Supplementary figure s7 shows serious arrhythmia incidence rates per day in the seven day current use period among fluoroquinolone users and penicillin $\mathrm{V}$ users. The absolute risk difference for current fluoroquinolone use compared with current penicillin $\mathrm{V}$ use, estimated as the number of cases of severe arrhythmia per 1000000 treatment courses, was -13 (95\% confidence interval -35 to 16 ). There was no increased risk associated with oral fluoroquinolone compared with penicillin $\mathrm{V}$ in periods of indeterminate use (rate ratio $1.13,0.71$ to 1.78 ) or past use (1.08, 0.83 to 1.42 , table 2$)$. 


\begin{tabular}{|c|c|c|c|}
\hline Characteristics & Fluoroquinolones & Penicillin V & Standardised difference \\
\hline No in cohort & 909656 & 909656 & \\
\hline \multicolumn{4}{|l|}{ Country: } \\
\hline Denmark & $309995(34.1)$ & $309995(34.1)$ & - \\
\hline Sweden & $599661(65.9)$ & $599661(65.9)$ & - \\
\hline Men & $487141(53.6)$ & $490199(53.9)$ & 0.007 \\
\hline Mean (SD) age (years) & $60.7(10.7)$ & $60.7(10.6)$ & 0.001 \\
\hline \multicolumn{4}{|l|}{ Calendar year: } \\
\hline $1997-99$ & $50272(5.5)$ & $50141(5.5)$ & 0.001 \\
\hline $2000-02$ & $34485(3.8)$ & $34252(3.8)$ & 0.001 \\
\hline 2003-05 & $52011(5.7)$ & $51663(5.7)$ & 0.002 \\
\hline 2006-08 & $343836(37.8)$ & $347723(38.2)$ & 0.009 \\
\hline 2009-11 & $303971(33.4)$ & $302381(33.2)$ & 0.004 \\
\hline $2012-13$ & $125081(13.8)$ & $123496(13.6)$ & 0.005 \\
\hline \multicolumn{4}{|l|}{ Periods: } \\
\hline March-May & $216932(23.8)$ & $216598(23.8)$ & 0.001 \\
\hline June-August & $216510(23.8)$ & $216513(23.8)$ & 0.000 \\
\hline September-November & $241599(26.6)$ & $241877(26.6)$ & 0.001 \\
\hline December-February & $234615(25.8)$ & $234668(25.8)$ & 0.000 \\
\hline \multicolumn{4}{|l|}{ Medical history: } \\
\hline Acute coronary syndrome & $26808(2.9)$ & $26818(2.9)$ & 0.000 \\
\hline Other ischaemic heart disease & $67752(7.4)$ & $67962(7.5)$ & 0.001 \\
\hline Heart failure/cardiomyopathy & $24804(2.7)$ & $24540(2.7)$ & 0.002 \\
\hline Valve disorders & $11295(1.2)$ & $11225(1.2)$ & 0.001 \\
\hline Cerebrovascular disease & 33189 (3.6) & $32314(3.6)$ & 0.005 \\
\hline Arterial disease & $25434(2.8)$ & $24724(2.7)$ & 0.005 \\
\hline Arrhythmia & $55201(6.1)$ & $55455(6.1)$ & 0.001 \\
\hline Renal disease & $13979(1.5)$ & $13351(1.5)$ & 0.006 \\
\hline Other respiratory disease & $56327(6.2)$ & $56572(6.2)$ & 0.001 \\
\hline Other psychiatric disorder & $41504(4.6)$ & $41442(4.6)$ & 0.000 \\
\hline Cardiac surgery/invasive procedure in past year & $9614(1.1)$ & $9606(1.1)$ & 0.000 \\
\hline \multicolumn{4}{|l|}{ Use of healthcare in previous six months: } \\
\hline Cardiovascular emergency visit/hospital admission & $66410(7.3)$ & $64191(7.1)$ & 0.009 \\
\hline Other emergency visit/hospital admission & $251658(27.7)$ & $247868(27.2)$ & 0.009 \\
\hline Cardiovascular outpatient contact & $46156(5.1)$ & $46154(5.1)$ & 0.000 \\
\hline Other outpatient contact & $321595(35.4)$ & $321313(35.3)$ & 0.001 \\
\hline Emergency visits in past 7 days & 35235 (3.9) & $33458(3.7)$ & 0.010 \\
\hline \multicolumn{4}{|l|}{ Prescription drug use in previous year: } \\
\hline Angiotensin receptor blocker/angiotensin converting enzyme inhibitor & $219863(24.2)$ & $220892(24.3)$ & 0.003 \\
\hline Dihydropyridine calcium channel blocker & $103684(11.4)$ & $103388(11.4)$ & 0.001 \\
\hline Non-dihydropyridine calcium channel blocker & $14631(1.6)$ & $14640(1.6)$ & 0.000 \\
\hline Loop diuretics & $72886(8.0)$ & $72568(8.0)$ & 0.001 \\
\hline Other diuretics & $109336(12.0)$ & $109186(12.0)$ & 0.001 \\
\hline$\beta$ blockers & $184701(20.3)$ & $185166(20.4)$ & 0.001 \\
\hline Anti-arrhythmic class I and III & $5131(0.6)$ & $5235(0.6)$ & 0.002 \\
\hline Digoxin & $13772(1.5)$ & $13750(1.5)$ & 0.000 \\
\hline Nitrates & $48657(5.3)$ & $48738(5.4)$ & 0.000 \\
\hline Platelet inhibitors & $166141(18.3)$ & $166356(18.3)$ & 0.001 \\
\hline Anticoagulants & $34739(3.8)$ & $34957(3.8)$ & 0.001 \\
\hline Lipid lowering drugs & $187517(20.6)$ & $188806(20.8)$ & 0.003 \\
\hline Oral antidiabetic drugs & $63478(7.0)$ & $63574(7.0)$ & 0.000 \\
\hline Insulin & $36517(4.0)$ & $36401(4.0)$ & 0.001 \\
\hline Antidepressants & $130600(14.4)$ & $129820(14.3)$ & 0.002 \\
\hline Antipsychotics & $22080(2.4)$ & $22210(2.4)$ & 0.001 \\
\hline Anxiolytics, hypnotics, and sedatives & $215226(23.7)$ & $215983(23.7)$ & 0.002 \\
\hline$\beta 2$ agonist inhalants & $79854(8.8)$ & $80042(8.8)$ & 0.001 \\
\hline Corticosteroid inhalants & $96144(10.6)$ & $96695(10.6)$ & 0.002 \\
\hline Xantines & $5057(0.6)$ & $5110(0.6)$ & 0.001 \\
\hline Anticholinergic inhalants & $25043(2.8)$ & $24984(2.7)$ & 0.000 \\
\hline Oral corticosteroids & $92834(10.2)$ & $93694(10.3)$ & 0.003 \\
\hline Non-steroidal anti-inflammatory drugs & $282665(31.1)$ & $283340(31.1)$ & 0.002 \\
\hline Opioids & $169024(18.6)$ & $168357(18.5)$ & 0.002 \\
\hline Systemic hormone replacement therapy & $118786(13.1)$ & $119718(13.2)$ & 0.003 \\
\hline Anti-osteoporotic drugs & $23948(2.6)$ & $24111(2.7)$ & 0.001 \\
\hline Drugs for peptic ulcer/gastroesophageal reflux & $208592(22.9)$ & $209157(23.0)$ & 0.001 \\
\hline \multicolumn{4}{|l|}{ No of prescription drugs used in previous year: } \\
\hline $0-4$ & $376232(41.4)$ & $373530(41.1)$ & 0.006 \\
\hline $5-9$ & $291144(32.0)$ & $293242(32.2)$ & 0.005 \\
\hline$>10$ & $242280(26.6)$ & $242884(26.7)$ & 0.002 \\
\hline
\end{tabular}




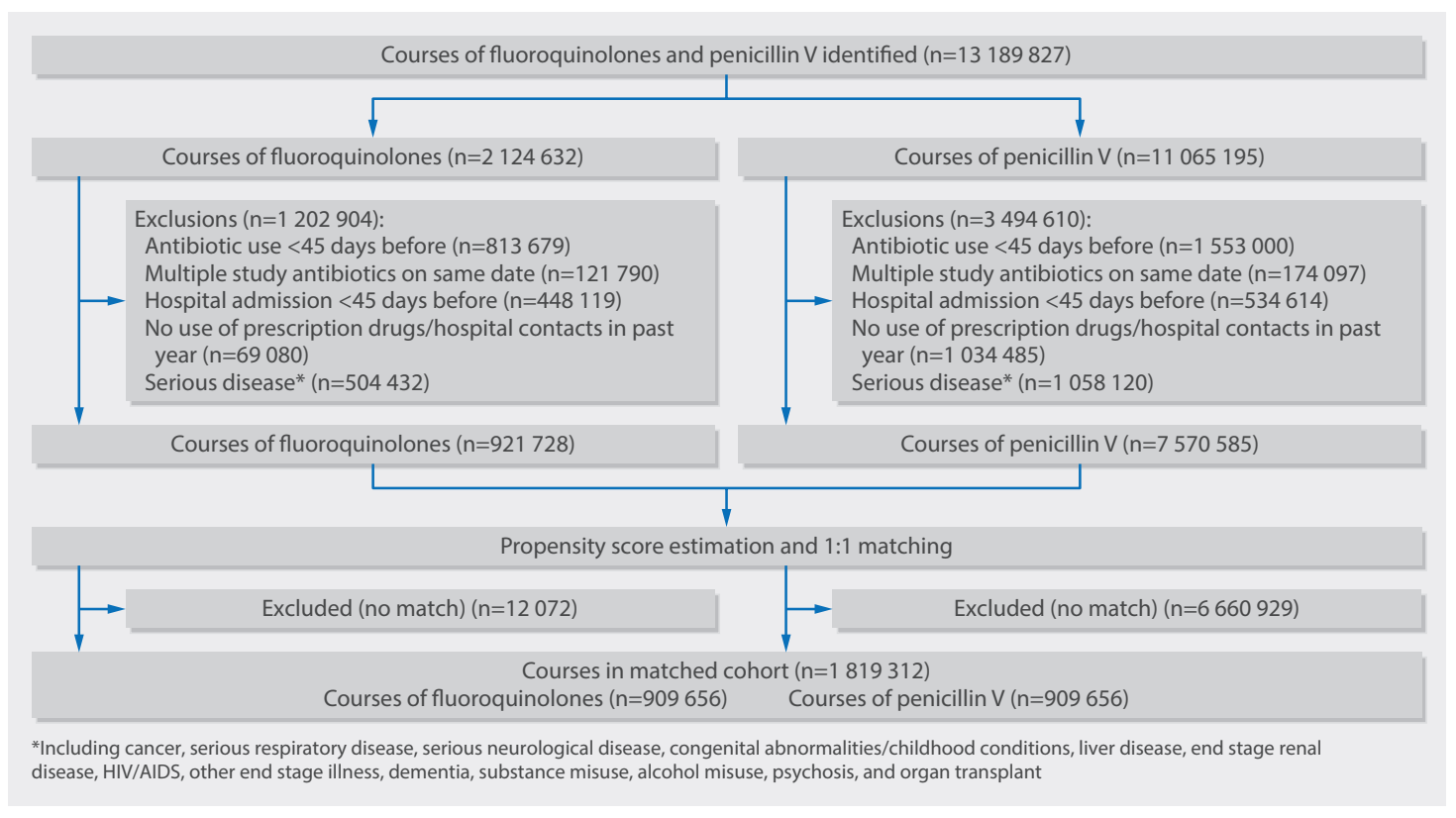

Fig 1 | Flow chart of cohort selection. Values for exclusion criteria do not sum to totals shown as some records were excluded for multiple reasons

\begin{tabular}{|c|c|c|c|}
\hline Drugs by period of use & $\begin{array}{l}\text { Cases of } \\
\text { arrhythmia }\end{array}$ & $\begin{array}{l}\text { Incidence } \\
\text { rate per } 1000 \\
\text { person years }\end{array}$ & Rate ratio $(95 \% \mathrm{Cl})$ \\
\hline \multicolumn{4}{|l|}{ Current use*: } \\
\hline Fluoroquinolones & 66 & 3.4 & 0.85 (0.61 to 1.18$)$ \\
\hline Penicillin V & 78 & 4.0 & 1.00 (reference) \\
\hline \multicolumn{4}{|l|}{ Indeterminate uset: } \\
\hline Fluoroquinolones & 39 & 2.5 & 1.13 (0.71 to 1.78$)$ \\
\hline Penicillin V & 35 & 2.2 & 1.00 (reference) \\
\hline \multicolumn{4}{|l|}{ Past useł: } \\
\hline Fluoroquinolones & 108 & 1.8 & 1.08 (0.83 to 1.42$)$ \\
\hline Penicillin V & 103 & 1.7 & 1.0 reference) \\
\hline
\end{tabular}

\section{Subgroup analyses}

Table 3 shows rate ratios of serious arrhythmia for current use of oral fluoroquinolones compared with penicillin $\mathrm{V}$ according to different subgroups. No statistically significant differences in rate ratios were observed according to underlying cardiovascular disease (test of homogeneity $\mathrm{P}=0.91$ ), co-treatment with drugs known to increase the risk of torsades de pointes $(\mathrm{P}=0.43)$, country of residence $(\mathrm{P}=0.42)$, or $\operatorname{sex}(\mathrm{P}=0.13)$, or across levels of arrhythmia risk score $(\mathrm{P}=0.66)$. There was a significant difference according to age group (rate ratio 0.45 (95\% confidence interval 0.23 to 0.88 ) among participants aged 40-64 years, and 1.07 (0.73 to 1.57) among participants aged 65-79 years, $\mathrm{P}=0.02)$. The risk associated with use of ciprofloxacin compared with penicillin $\mathrm{V}$ was not significantly different from the risk associated with all other fluoroquinolones combined $(\mathrm{P}=0.61)$.

\section{Secondary analyses}

Sensitivity analyses using only the first antibiotic course yielded similar results and did not change the conclusions of no observed risk of serious arrhythmia in current users of oral fluoroquinolones compared with penicillin V (rate ratio 1.02, 95\% confidence interval 0.63 to 1.65$)$. We observed no increased risk when restricting the analyses to fatal arrhythmic events only, neither in the period of current use (1.07, 0.54 to 2.12) nor in periods of indeterminate or past use. We did not observe an increased risk of mortality from any cause other than serious arrhythmia (rate ratio for current use $0.95,0.83$ to 1.09 ).

\section{Discussion}

In this large cohort study of a general adult population from two countries, comprising more than 900000 treatment courses with fluoroquinolones, we did not find an increased risk of serious arrhythmia compared with use of penicillin V. Given the upper limit of the confidence interval, we could rule out an increased relative risk of $18 \%$ of serious arrhythmia. Correspondingly, in absolute terms, any residual risk would account for at most 16 cases per 1000000 treatment courses. Reassuringly, our results were homogenous and we did not observe increased risks in groups of participants with underlying cardiovascular disease or who concurrently used drugs that increase the risk of torsades de pointes. The relative risk in the younger age group was significantly lower; however, since the rate of serious arrhythmia was markedly lower with lower age, the analysis was based on few events in the younger subgroup.

\section{Strengths and limitations of this study}

Our study has several strengths. Firstly, the use of nationwide cohorts from two different countries assures that our results are generalisable to a general adult population. Secondly, we used multiple strategies to reduce confounding. We used propensity score methods to balance the treatment groups on a large range of baseline 


\begin{tabular}{|c|c|c|c|c|c|c|}
\hline \multirow[b]{2}{*}{ Analyses } & \multicolumn{2}{|c|}{ Fluoroquinolones } & \multicolumn{2}{|l|}{ Penicillin V } & \multirow[b]{2}{*}{$\begin{array}{l}\text { Rate ratio }(95 \% \\
\mathrm{Cl})\end{array}$} & \multirow[b]{2}{*}{ Pvalue $^{\dagger}$} \\
\hline & $\begin{array}{l}\text { Cases of } \\
\text { arrhythmia }\end{array}$ & $\begin{array}{l}\text { Incidence rate } \\
\text { per } 1000 \\
\text { person years }\end{array}$ & $\begin{array}{l}\text { Cases of } \\
\text { arrhythmia }\end{array}$ & $\begin{array}{l}\text { Incidence rate } \\
\text { per } 1000 \\
\text { person years }\end{array}$ & & \\
\hline Primary analysis & 66 & 3.4 & 78 & 4.0 & 0.85 (0.61 to 1.18$)$ & \\
\hline \multicolumn{7}{|l|}{ Sex: } \\
\hline Women & 23 & 2.6 & 18 & 2.0 & 1.27 (0.69 to 2.36$)$ & \multirow{2}{*}{0.13} \\
\hline Men & 43 & 4.2 & 60 & 5.8 & 0.73 (0.49 to 1.08$)$ & \\
\hline \multicolumn{7}{|l|}{ Age (years): } \\
\hline $40-64$ & 12 & 1.0 & 27 & 2.3 & $0.45(0.23$ to 0.88$)$ & \multirow{2}{*}{0.02} \\
\hline $65-80$ & 54 & 7.5 & 51 & 7.0 & 1.07 (0.73 to 1.57$)$ & \\
\hline \multicolumn{7}{|c|}{ History of cardiovascular disease: } \\
\hline No & 26 & 1.6 & 30 & 1.9 & 0.87 (0.52 to 1.47) & \multirow{2}{*}{0.91} \\
\hline Yes & 40 & 12.7 & 48 & 15.1 & $0.84(0.55$ to 1.28$)$ & \\
\hline \multicolumn{7}{|c|}{ Co-treatment with drug for torsades de pointes: } \\
\hline No & 54 & 3.0 & 60 & 3.3 & 0.91 (0.63 to 1.31) & \multirow{2}{*}{0.43} \\
\hline Yes & 12 & 12.3 & 18 & 18.8 & $0.65(0.31$ to 1.38$)$ & \\
\hline \multicolumn{7}{|l|}{ Country of residence: } \\
\hline Denmark & 30 & 4.6 & 30 & 4.6 & $1.00(0.60$ to 1.65$)$ & \multirow{2}{*}{0.42} \\
\hline Sweden & 36 & 2.9 & 48 & 3.8 & $0.76(0.49$ to 1.17$)$ & \\
\hline \multicolumn{7}{|l|}{ Fluoroquinolone: } \\
\hline Ciprofloxacin & 56 & 3.5 & 78 & 4.0 & 0.88 (0.62 to 1.24) & \multirow{2}{*}{0.61} \\
\hline All other fluoroquinolones & 10 & 3.0 & 78 & 4.0 & $0.74(0.38$ to 1.43$)$ & \\
\hline \multicolumn{7}{|l|}{ Arrythmia risk scoreł: } \\
\hline Low & 3 & 0.3 & 6 & 0.6 & 0.50 (0.13 to 2.00$)$ & \multirow{3}{*}{0.66} \\
\hline Moderate & 9 & 1.6 & 11 & 1.9 & 0.83 (0.34 to 2.01) & \\
\hline High & 54 & 14.2 & 61 & 15.9 & 0.89 (0.61 to 1.29$)$ & \\
\hline
\end{tabular}

*Days 0-7 after prescription.

†Test of homogeneity.

‡Categorised into three levels (by 10ths): low (1-5), moderate (6-8) and high (9-10).

health factors. Additionally, we excluded participants with end stage serious disease or substance misuse to reduce the numbers of people at high risk irrespective of fluoroquinolone use. We also excluded those with previous antibiotic prescriptions or hospital admissions, and censored those with subsequent antibiotic prescriptions or hospital admissions to minimise misclassification of drug use. Finally, we utilised a comparative design with a reference antibiotic without pro-arrhythmic effect, to minimise confounding by indication.

A limitation of the study is the lack of information on the actual duration of treatment. This was accounted for by inclusion of the risk period of indeterminate use. Another limitation is the lack of information on the indication for treatment, as well as information on other important risk factors for ventricular arrhythmia (eg, electrolyte disturbances and degree of organic heart disease), therefore residual confounding cannot be ruled out despite multiple measures to minimise confounding. However, the robustness of our results is supported by the fact that the rates of serious arrhythmia were not significantly different in fluoroquinolone and penicillin $\mathrm{V}$ users in the period of past use, which was considered to mirror the baseline risk of serious arrhythmia.

The comparator antibiotic penicillin V, the most commonly used antibiotic in both Denmark and Sweden, has no known pro-arrhythmic effect. Indications overlap for the fluoroquinolones with effect on Gram positive bacteria, such as ofloxacin, moxifloxacin, and levofloxacin, with regard to respiratory infections, but older fluoroquinolones such as norfloxacin and ciprofloxacin, with predominate effect on Gram negative pathogens, are mainly used in gastrointestinal and urinary tract infections. However, even though the indications for treatment (type of the acute infection) did not overlap perfectly, the mortality due to other causes did not differ between fluoroquinolone and penicillin $\mathrm{V}$ users in our study. This indicates an equal degree of severity of the acute infections for which the treatment were prescribed, as well as similar heath status at baseline. We therefore believe penicillin $\mathrm{V}$ is a valid comparator.

The outcome "serious arrhythmia" as defined in the current study, has not been formally validated. However, in previous studies of myocardial infarction, cardiac death, and atrial fibrillation, $86-97 \%$ of diagnoses from the Danish patient register and causes of death register, were correct according to the original medical or autopsy files ${ }^{25-27}$; similarly, in Sweden validation studies suggests that $90-100 \%$ of these diagnoses are correct. ${ }^{1928}$

Non-fatal ventricular arrhythmia is likely to be underdiagnosed, ${ }^{1}$ and misclassification could be differential (higher ascertainment in fluoroquinolone users) owing to a presumed increased risk of QT prolongation. If present, this bias would inflate estimates, while our finding of no association would still hold true.

\section{Comparison with other studies}

Despite the potential for prolongation of the QT interval during fluoroquinolone treatment, few studies have 
explored the association with arrhythmia in a larger clinical setting. A case-control study from Italy reported a fourfold increased risk of serious arrhythmia within four weeks of fluoroquinolone use. ${ }^{10}$ A nested case-control study from Canada reported a twofold increased risk within two weeks of fluoroquinolone use. ${ }^{9}$ Both of these studies, however, assessed the risk associated with fluoroquinolone use compared with no use of antibiotics. Consequently, it cannot be ruled out that the reported increased risks were attributable to the indication for treatment-that is, the acute infections. An acute infection can facilitate arrhythmia, cardiac arrest, and sudden death through a wide range of mechanisms, such as hypovolaemia, disturbance of electrolytes, acute kidney failure, and aggravated heart failure. ${ }^{29}$

In a cohort study of US veterans, a 2.5-fold increased risk of serious arrhythmia was reported among users of levofloxacin compared with amoxicillin. Notably, however, a 2.5-fold increased risk of all cause mortality was also observed with levofloxacin. This noticeable difference in all cause mortality implies important differences in health status between those treated with fluoroquinolones and those treated with amoxicillin, which may also have confounded the estimate for serious arrhythmia. ${ }^{12}$ In line with our findings, although using a broader outcome definition, no significantly increased risk of cardiovascular death associated with ciprofloxacin compared with amoxicillin was observed in a cohort study of Medicaid beneficiaries. However, in this study, the hazard ratio for cardiovascular death associated with levofloxacin was 1.50 (95\% confidence interval 0.82 to 2.72$){ }^{30}$

Recently, a cohort study from Taiwan reported a threefold significantly increased odds ratio for ventricular arrhythmia and cardiovascular death with use of moxifloxacin, but not ciprofloxacin or levofloxacin, compared with amoxicillin-clavulanate. ${ }^{11}$ The observed association with use of moxifloxacin, however, persisted in periods of up to 30 days after the start of treatment, which implies that factors other than treatment itself, such as baseline health of the participants or the severity of infection, may have influenced the results. All cause mortality and other causes of mortality were not reported.

Experimental studies and small trials in humans suggest that the potential for cardiac potassium channel inhibition may differ slightly between the various fluoroquinolone agents; eg, moxifloxacin has been associated with a slightly greater effect on prolongation of the QT interval. ${ }^{3513}$ However, results are somewhat conflicting and it is not established whether these differences translate to differential risks of serious arrhythmia in a real world clinical setting. ${ }^{14}$ Additional pro-arrhythmic mechanisms have been described for fluoroquinolones, such as triangulation of the action potential and dispersion of repolarisation. ${ }^{31}$ Among low potency blockers of the cardiac potassium channel such as fluoroquinolones, QT studies may not be precise enough to predict any differences in pro-arrhythmogenic potential between agents, and other factors that alter drug concentrations, such as impaired drug clearance, may be more important than specific drug related risk factors. ${ }^{32}$

The effect on cardiac rhythm of levofloxacin and moxifloxacin was assessed in a randomised clinical study of patients aged 65 years or more with pneumonia. The authors found no correlation between prolongation of the QT interval and cardiac events and no significant difference between the two treatment arms. ${ }^{33}$ Most treatment courses with fluoroquinolones in our study were with ciprofloxacin, thus our results are primarily applicable to this fluoroquinolone. We did not find statistical evidence of heterogeneity between ciprofloxacin and other fluoroquinolones, but we cannot exclude the possibility that the specific agents in this drug class might increase the risk of serious arrhythmia differentially.

\section{Conclusions and policy implications}

We cannot exclude that in some situations fluoroquinolones can cause torsades de pointes. However, we did not find support for an increased risk of serious arrhythmia associated with oral fluoroquinolone use in a general adult outpatient population. Among those with severe infections requiring hospital stay, further research is needed to assess the magnitude of the pro-arrhythmic potential of fluoroquinolones. Since ciprofloxacin was the most commonly used fluoroquinolone in our study we cannot exclude that intraclass differences influence the risk of serious arrhythmias associated with other fluoroquinolones.

Contributors: All authors conceived and designed the study and analysed and interpreted the results. HS and MI acquired data and conducted the statistical analyses. MI, HS, and BJP drafted the manuscript. All authors critically revised the manuscript and approved the final version for submission. AH supervised the study and is the guarantor.

Funding: This study was supported by grants from the Swedish Government Funds for Clinical Research (ALF), Scandinavian Society for Antimicrobial Chemotherapy Foundation, (SSAC), Royal Physiographic Society in Lund, and Danish Council for Independent Research (Medical Sciences). The funders played no role in the design of the study, data collection or analysis, decision to publish, or preparation of the manuscript.

Competing interests: All authors have completed the ICMJE uniform disclosure form at www.icmje.org/coi_disclosure.pdf (available on request from the corresponding author) and declare: no financial relationships with any organization for the submitted work; no financial relationship with any organisations that might have an interest in the submitted work in the previous three years; no other relationships or activities that could appear to have influenced the submitted work.

Ethical approval: The Danish Data Protection Agency approved the Danish part of the study. Ethical approval is not required for register based research in Denmark. The local ethics committee in Lund (Dnr: 2013/717) approved the Swedish parts of the study.

Data sharing: No additional data available.

Transparency: The manuscript's guarantor (AH) affirms that this manuscript is an honest, accurate, and transparent account of the study being reported; that no important aspects of the study have been omitted; and that any discrepancies from the study as planned have been explained.

This is an Open Access article distributed in accordance with the Creative Commons Attribution Non Commercial (CC BY-NC 3.0) license, which permits others to distribute, remix, adapt, build upon this work non-commercially, and license their derivative works on different terms, provided the original work is properly cited and the use is non-commercial. See: http://creativecommons.org/licenses/ by-nc/3.0/. 
1 Owens RC Jr, , Nolin TD. Antimicrobial-associated QT interval prolongation: pointes of interest. Clin Infect Dis 2006;43:1603-11. doi:10.1086/508873.

2 Jaillon P, Morganroth J, Brumpt I, et al. Overview of electrocardiographic and cardiovascular safety data for sparfloxacin. Sparfloxacin Safety Group. I Antimicrob Chemother 1996;37 Suppl A:161-7.

3 Tsikouris JP, Peeters MJ, Cox CD, Meyerrose GE, Seifert CF. Effects of three fluoroquinolones on QT analysis after standard treatment courses. Ann Noninvasive Electrocardiol 2006;11:52-6. doi:10.1111/j.1542-474X.2006.00082.x.

4 Makaryus AN, Byrns K, Makaryus MN, Natarajan U, Singer C, Goldner B. Effect of ciprofloxacin and levofloxacin on the QT interval: is this a significant "clinical" event?South Med/ 2006;99:52-6. doi:10.1097/01.smi.0000197124.31174.7e.

5 Noel GJ, Natarajan J, Chien S, Hunt TL, Goodman DB, Abels R. Effects of three fluoroquinolones on QT interval in healthy adults after single doses. Clin Pharmacol Ther 2003;73:292-303. doi:10.1016/ S0009-9236(03)00009-2.

6 Frothingham R. Rates of torsades de pointes associated with ciprofloxacin ofloxacin, levofloxacin gatifloxacin, and moxifloxacin. Pharmacotherapy 2001;21:1468-72. doi:10.1592/ phco.21.20.1468.34482.

7 Poluzzi E, Raschi E, Motola D, Moretti U, De Ponti F. Antimicrobials and the risk of torsades de pointes: the contribution from data mining of the US FDA Adverse Event Reporting System. Drug Saf 2010;33:30314. doi:10.2165/11531850-000000000-00000.

8 Clark DW, Layton D, Wilton LV, Pearce GL, Shakir SA. Profiles of hepatic and dysrhythmic cardiovascular events following use of fluoroquinolone antibacterials: experience from large cohorts from the Drug Safety Research Unit Prescription-Event Monitoring database. Drug Saf 2001:24:1143-54 doi:10.2165/00002018-200124150-00005.

9 Lapi F, Wilchesky M, Kezouh A, Benisty Jl, Ernst P, Suissa S. Fluoroquinolones and the risk of serious arrhythmia: a populationbased study. Clin Infect Dis 2012;55:1457-65. doi:10.1093/cid/ cis664.

10 Zambon A, Polo Friz H, Contiero P, Corrao G. Effect of macrolide and fluoroquinolone antibacterials on the risk of ventricular arrhythmia and cardiac arrest: an observational study in Italy using case-control, case-crossover and case-time-control designs. Drug Saf 2009;32:159-67. doi:10.2165/00002018-200932020-00008.

11 Chou HW, Wang JL, Chang CH, Lai CL, Lai MS, Chan KA. Risks of cardiac arrhythmia and mortality among patients using new-generation macrolides, fluoroquinolones, and $\beta$-lactam/ $\beta$-lactamase inhibitors: a Taiwanese nationwide study. Clin Infect Dis 2015;60:566-77. doi:10.1093/cid/ciu914

12 Rao GA, Mann JR, Shoaibi A, et al. Azithromycin and levofloxacin use and increased risk of cardiac arrhythmia and death. Ann Fam Med 2014;12:121-7. doi:10.1370/afm.1601.

13 Anderson ME, Mazur A, Yang T, Roden DM. Potassium current antagonist properties and proarrhythmic consequences of quinolone antibiotics. J Pharmacol Exp Ther 2001;296:806-10.

14 Abo-Salem E, Fowler JC, Attari M, et al. Antibiotic-induced cardiac arrhythmias. Cardiovasc Ther 2014;32:19-25. doi:10.1111/1755-5922.12054.

15 www.crediblemeds.org. Secondary.

16 Kildemoes HW, Sørensen HT, Hallas J. The Danish National Prescription Registry. Scand I Public Health 2011;39(Suppl):38-41. doi:10.1177/1403494810394717.

17 Wettermark B, Hammar N, Fored CM, et al. The new Swedish Prescribed Drug Register--opportunities for pharmacoepidemiological research and experience from the first six months. Pharmacoepidemiol Drug Saf 2007;16:726-35. doi:10.1002/ pds.1294.
18 Lynge E, Sandegaard JL, Rebolj M. The Danish National Patient Register. Scand J Public Health 2011;39(Suppl):30-3. doi:10.1177/1403494811401482.

19 Ludvigsson JF, Andersson E, Ekbom A, et al. External review and validation of the Swedish national inpatient register. BMC Public Health 2011:11:450. doi:10.1186/1471-2458-11-450.

20 Helweg-Larsen K. The Danish Register of Causes of Death. Scand Public Health 2011;39(Suppl):26-9. doi:10.1177/1403494811399958.

21 Glynn RJ, Schneeweiss S, Stürmer T. Indications for propensity scores and review of their use in pharmacoepidemiology. Basic Clin Pharmacol Toxicol 2006;98:253-9. doi:10.1111/j.1742-7843.2006.pto_293.x.

22 Rassen JA, Shelat AA, Franklin JM, Glynn RJ, Solomon DH, Schneeweiss S. Matching by propensity score in cohort studies with three treatment groups. Epidemiology 2013;24:401-9. doi:10.1097/ EDE.0b013e318289dedf

23 Parson L. Reducing bias in a propensity score matched-pair sample using greedy matching techniques. Proceedings of the 26th Annual SAS Users Group International Conference, SAS 2001:214-6.

24 Stürmer T, Schneeweiss S, Brookhart MA, Rothman KJ, Avorn J, Glynn RJ. Analytic strategies to adjust confounding using exposure propensity scores and disease risk scores: nonsteroidal antiinflammatory drugs and short-term mortality in the elderly. Am J Epidemiol 2005;161:891-8. doi:10.1093/aje/kwi106.

25 Frost L, Vestergaard P. Alcohol and risk of atrial fibrillation or flutter: a cohort study. Arch Intern Med 2004;164:1993-8. doi:10.1001/ archinte.164.18.1993.

26 Madsen M, Davidsen M, Rasmussen S, Abildstrom SZ, Osler M. The validity of the diagnosis of acute myocardial infarction in routine statistics: a comparison of mortality and hospital discharge data with the Danish MONICA registry. J Clin Epidemiol 2003;56:124-30. doi:10.1016/S0895-4356(02)00591-7.

27 Winkel BG, Holst AG, Theilade J, et al. Nationwide study of sudden cardiac death in persons aged 1-35 years. Eur Heart J 2011;32:98390. doi:10.1093/eurheartj/ehq428.

28 Smith JG, Platonov PG, Hedblad B, Engström G, Melander O. Atrial fibrillation in the Malmö Diet and Cancer study: a study of occurrence, risk factors and diagnostic validity. Eur J Epidemiol 2010;25:95-102. doi:10.1007/s10654-009-9404-1.

29 Justo D, Zeltser D. Torsade de pointes induced by systemic antifungal agents: lessons from a retrospective analysis of published case reports. Mycoses 2006;49:463-70. doi:10.1111/j.1439-0507.2006.01278.x.

30 Ray WA, Murray KT, Hall K, Arbogast PG, Stein CM. Azithromycin and the risk of cardiovascular death. N Engl J Med 2012;366:1881-90. doi:10.1056/NEJMoa1003833.

31 Milberg P, Hilker E, Ramtin S, et al. Proarrhythmia as a class effect of quinolones: increased dispersion of repolarization and triangulation of action potential predict torsades de pointes. J Cardiovasc Electrophysiol 2007:18:647-54 doi:10.1111/j.1540-8167.2007.00793.x

32 Redfern WS, Carlsson L, Davis AS, et al. Relationships between preclinical cardiac electrophysiology, clinical QT interval prolongation and torsade de pointes for a broad range of drugs: evidence for a provisional safety margin in drug development. Cardiovasc Res 2003:58:32-45. doi:10.1016/S0008-6363(02)00846-5.

33 Morganroth J, Dimarco JP, Anzueto A, Niederman MS, Choudhri S. CAPRIE Study Group. A randomized trial comparing the cardiac rhythm safety of moxifloxacin vs levofloxacin in elderly patients hospitalized with community-acquired pneumonia. Chest 2005;128:3398-406. doi:10.1378/chest.128.5.3398.

Supplementary appendix: additional information 